\title{
Galectin-1-mediated cell death is increased by CD30-induced signaling in anaplastic large cell lymphoma cells but not in Hodgkin lymphoma cells
}

\author{
Osamu Suzuki ${ }^{1,2,4}$, Burkhard Hirsch ${ }^{1,4}$, Masafumi Abe ${ }^{2}$, Horst Dürkop $^{3}$ and Harald Stein ${ }^{3}$
}

\begin{abstract}
Endogenous $\beta$-galactose-binding lectins have many biological functions, but their biological significance in Hodgkin lymphoma $(\mathrm{HL})$ and anaplastic large cell lymphoma $(\mathrm{ALCL})$ remains unclear. By immunohistochemistry, we analyzed the expression of galectin-1 and galectin-3 in HL and ALCL cases as well as in cell lines, and investigated the pharmacological effects of galectin-1 treatment with and without CD30 pre-stimulation of $\mathrm{HL}$ and $\mathrm{ALCL}$ cell lines. The galectin-3-negative human embryonic kidney cell line (HEK-293) was transfected with galectin-3 cDNA. Galectin-3 is differentially expressed in $\mathrm{HL}$ and ALCL. CD30 stimulation of the ALCL cell line Karpas 299 activates NF- $\kappa$ B without induction of apoptosis. Galectin-1 treatment of Karpas 299 induces cell death, which is significantly increased by CD30 pre-stimulation. The CD30-mediated increase of galectin-1-induced cell death is to some extent caspase independent and does not influence the expression of tumor necrosis factor-associated factor 1 (TRAF1), TRAF2, and cellular inhibitor of apoptosis 2 protein (cIAP2), as revealed in Karpas 299 cells. In other cell lines except Karpas 299, CD30 pre-stimulation did not significantly enhance galectin-1-induced cell death. Galectin-3 transfection of HEK-293 cells resulted in cell surface expression of galectin-3, associated with marked cell aggregation. CD30-targeted therapy in combination with galectin-1 treatment may induce effective killing of ALCL cells but not of HL cells.

Laboratory Investigation (2012) 92, 191-199; doi:10.1038/labinvest.2011.151; published online 10 October 2011
\end{abstract}

KEYWORDS: anaplastic large cell lymphoma; CD30; cell death; galectin-1; galectin-3; Hodgkin lymphoma; lectins

Galectin is a member of the family of endogenous mammalian $\beta$-galactoside-binding lectins that appears to be involved in a variety of biological phenomena. ${ }^{1,2}$ Galectin regulates cell growth ${ }^{3}$ and apoptosis. ${ }^{4}$ Galectin-1 is a useful marker for differentiation between classical Hodgkin lymphoma (cHL) and nodular lymphocyte predominant Hodgkin lymphoma ${ }^{5}$ or between cHL and diffuse large B-cell lymphoma (DLBCL). ${ }^{6}$ In addition, alterations of N-glycans on the cell surface can regulate cell death in DLBCL by interacting with galectin-1.

CD30 is a member of the TNF receptor family, which is almost selectively expressed on the Hodgkin-Reed-Sternberg (HRS) cells of cHL as well as the tumor cells of anaplastic large cell lymphoma (ALCL), DLBCL of the anaplastic variant, and embryonal carcinoma of the testis. CD30 lacks an intracellular death domain. Stimulation of CD30 leads to
NF- $\kappa \mathrm{B}$ activation and the induction of anti-apoptotic mechanisms. $^{8}$

Galectin-3 is expressed in ALCL and is a useful marker for differentiation between ALCL and $\mathrm{CHL}^{9}$, as well as for differentiation between DLBCL and Burkitt's lymphoma. ${ }^{10}$ Galectin-3 represents a novel anti-apoptotic molecule containing the bcl-2 family $\mathrm{BH} 1$ domain that inhibits cytochrome c release from mitochondria. ${ }^{11}$ In contrast, secreted extracellular galectin-3 can induce apoptosis through the interaction with terminal galactose residues of cell surface glycans. ${ }^{12}$ We reported that alterations of $\mathrm{N}$-glycans and cell surface sialic acid can regulate galectin-3-induced apoptosis in DLBCL, which is consistent with the finding that $\mathrm{N}$-glycans and cell surface sialic acid represent one of the molecules that are associated with tolerance to galectin-3induced apoptosis of DLBCL. ${ }^{13}$ Hahn et al. ${ }^{14}$ reported that

\footnotetext{
${ }^{1}$ Institute of Pathology, Charité-University Medicine Berlin Campus Benjamin Franklin, Berlin, Germany; ${ }^{2}$ Department of Pathology and Diagnostic Pathology, School of Medicine, Fukushima Medical University, Fukushima, Japan and ${ }^{3}$ Pathodiagnostik, Berlin, Germany

Correspondence: Dr O Suzuki MD, PhD, Department of Pathology and Diagnostic pathology, School of Medicine, Fukushima Medical University, Hikariga-oka, Fukushima 960-1295, Japan.

E-mail: osuzuki@fmu.ac.jp

${ }^{4}$ These authors contributed equally to this work.

Received 7 April 2011; revised 5 August 2011; accepted 10 August 2011
} 
galectin-3 can inhibit galectin-1-induced apoptosis in Jurkat cell line. Noteworthy, in CHL and ALCL, the biological function of galectin-1 and galectin-3 remains unclear. We analyze the expression pattern of galectin- 1 and galectin- 3 in cHL and ALCL by immunohistochemistry, and investigated the pharmacological effects of galectin-1 treatment in combination with and without $\mathrm{CD} 30$ stimulation of $\mathrm{cHL}$ and ALCL cell lines.

\section{MATERIALS AND METHODS Tissue Microarray}

To prepare tissue microarrays (TMAs), tissue cores ( $1 \mathrm{~mm}$ in diameter) were removed from representative areas of the FFPE blocks of all samples and inserted into a recipient paraffin block. Sections were cut from the newly assembled TMA and used for immunohistochemical analysis. The TMA was prepared from 6 cases of cHL and 19 cases of ALCL. In addition, 10 ALCL cell lines (Mac-1, Mac-2a, Mac-2b, JK, FE-PD, SupM2, Karpas 299, JB6, SU-DHL1, and SR786) and 4 HL cell lines (L1236, HDLM2, KMH2, and L540) were included.

\section{Immunohistochemical Stainings}

Immunocytochemistry was carried out as published. ${ }^{9}$ In brief, immunohistologic stainings using galectin-1, AF1152, and galectin-3 from R\&D Systems (Wiesbaden, Germany) as primary antibodies were performed on TMA sections. Before incubation with the primary antibody, the dewaxed sections were subjected to an antigen retrieval protocol consisting of a brief, high-temperature heating of the sections immersed in a citrate buffer (10 mM, pH 6.0) for $2 \mathrm{~min}$ in a high-pressure cooker. Bound antibodies were made visible using the streptavidin biotin-alkaline phosphatase method and FastRed as chromogen. All incubations were performed in an automated immunostainer (TechMate 500 Dako). All reagents for that purpose were purchased from DAKO. Results were evaluated as follows: cases with $>30 \%$ positive tumor cells were graded positive and cases with $<30 \%$ of positive tumor cells were graded negative.

\section{Cell Lines and Treatments}

We used the B cell-derived HL cell lines, L1236, L428, KMH2 and the T cell-derived HL cell line HDLM2. The ALCL cell line, Karpas 299 was obtained from German Collection of Microorganisms and Cell Cultures (DSMZ, Braunschweig, Germany). The ALCL cell lines JB6 and FE-PD were generous gifts from Dr ME Kadin (Harvard Medical School, Boston, MA) and Dr K Pulford (John Radcliff Hospital, University of Oxford, Oxford, UK), respectively. The cell lines Karpas 299, JB6 are $t(2 ; 5)$ positive. Cells were maintained in RPMI-1640 with glutamine ( $4 \mathrm{mM}$; Gibco-BRL, Eggenstein, Germany) and $10 \%(\mathrm{v} / \mathrm{v})$ heat-inactivated fetal calf serum at $5 \% \mathrm{CO}_{2}$, $37^{\circ} \mathrm{C}$. All HL and ALCL cell lines express wild-type CD30 (B Hirsch, unpublished data).

Exponentially growing cells $\left(1.5 \times 10^{5} / \mathrm{ml}\right)$ were treated with CD30 ligand ( $100 \mathrm{ng} / \mathrm{ml}, \mathrm{R} \& \mathrm{D}$ Systems), CD30 agonistic murine monoclonal antibody (mAb) Ki-1 $(500 \mathrm{ng} / \mathrm{ml}, \operatorname{IgG} 3$ subclone produced in our institute), isotype-specific control $\mathrm{mAb} \quad\left(\mathrm{IgG}_{3}, 500 \mathrm{ng} / \mathrm{ml}\right.$, FLOPC 21, Sigma, Taufkirchen, Germany), or without any antibody for $24 \mathrm{~h}$. The recombinant $\mathrm{CD} 30$ ligand and soluble CD30 mAb (Ki-1) induced indistinguishable effects, as revealed by Reverse TranscriptionRelative Quantitative Real-Time PCR (RT-RQ-PCR) analysis of CD30-stimulated ALCL cell lines (data not shown). Therefore, we performed CD30 stimulation experiments of HL and ALCL cell lines with soluble Ki-1 or isotype $\operatorname{IgG}_{3}$ for control treatments, herein referred to as non-stimulated.

Cells were galectin- 1 treated ( $5 \mu \mathrm{M}$ bovine splenic galaptin, Sigma) or control treated $(0.05 \mathrm{M}$ Tris $)$ for $24 \mathrm{~h}$. For co-stimulation experiments, cells were CD30 pre-stimulated and galectin-1 treated. Induction of cell death was analyzed by FACS. The $5 \mu \mathrm{M}$ concentration of galectin- 1 was applied, because the concentration of galectin-1 to induce cell death is ranging from 4 to $16 \mu \mathrm{M}$ as previously described. ${ }^{4}$

We performed western blot analysis of tumor necrosis factor-associated factor 1 (TRAF1), TRAF2, and cellular inhibitor of apoptosis 2 protein (cIAP2) of CD30/controltreated Karpas 299 cells as well as Karpas 299 cells that have been CD30 pre-stimulated for $24 \mathrm{~h}$, followed by $5 \mu \mathrm{M}$ galectin- 1 treatment for $6 \mathrm{~h}$, by immunoblot.

The pan-caspase inhibitor benzyloxycarbonyl-Val-Ala-Asp (OMe) fluoromethylketone (Z-VAD-fmk) (Calbiochem, Schwalbach, Germany) was used at $50 \mu \mathrm{M}$ with a preincubation of $3 \mathrm{~h}$.

\section{Immunoblot Analysis}

Cell extracts $(24 \mu \mathrm{g})$ were fractioned on $4-12 \%(\mathrm{w} / \mathrm{v})$ SDSpolyacrylamide gels and transferred onto PVDF membranes (Amersham, Braunschweig, Germany). Immunoblot analysis was performed by TRAF2 (BD Pharmingen, dilution 6:1000), cIAP2 (Abcam, Cambridge, UK, ab32059, dilution 1:1000), TRAF 1 (produced in the Institute of Pathology, Charité Berlin, dilution 1:50), $\beta$-actin (Abcam), and galectin-3 (MAB1154, R\&D Systems). Primary antibodies were detected by HRP-conjugated antibodies and chemiluminescence (Amersham).

\section{Flow-Cytometric Analysis}

Staining of dead/apoptotic cells was performed by Annexin V-FITC Apoptosis Detection Kit I (BD Biosciences Pharmingen, Wiesbaden-Nordenstadt, Germany) according to the manufacturer's instructions. Galectin-3 is detected by galectin-3 antibody (R\&D Systems) and second anti-mouse FITC-conjugated antibody (DakoCytomation). Labeled cells were analyzed by FACSort (Becton Dickinson, Heidelberg, Germany).

\section{Knockdown of Galectin-3 by siRNA}

Galectin-3 siRNA (Dharmacon, ON-TARGETplus, No. L-010606-00, Target sequence J-010606-06: GGAGAGUCAU UGUUUGCAA, J-010606-07: GUACAAUCAUCGGGUUAAA, 
J-010606-08: GGCCACUG AUUGUGCCUUA, J-010606-09: CGGUGAAGCCCAAUGCAAA, USA) or negative control siRNA (Dharmacon, ON-TARGET plus siCONTROL, Nontargeting pool, D-001810-10, USA) was transfected by the nucleofector technology (Amaxa, Colonge, Germany) to Karpas 299 cell line. Transfection condition: $16 \mathrm{nM}$ siRNA, Nucleofector kit T (No. VCA-1002, Amaxa), program V-01. Protein and RNA analysis was performed as described. ${ }^{8}$

\section{RNA Extraction and RT-RQ-Real Time PCR}

RT-RQ-PCR was carried out as published. ${ }^{8}$ The following pairs of oligonucleotides (forward/reverse) were used: galectin-3 5'-CAATTCTGGGCACGGTGAA-3' $/ 5^{\prime}$-CATCATT CCCTCTTTGGAAATCTAA- $3^{\prime}$, Hypoxanthine phosphoribosyltransferase (HPRT) 5'-GAGTCCTATTGACATCGCCAG TA- $3^{\prime} / 5^{\prime}$-CTAAGCAGATGGCCACAGAACTAG-3' served as endogenous control. HPRT-normalized gene expression was calculated by the $2^{-\Delta \Delta}$ CT method. ${ }^{15}$

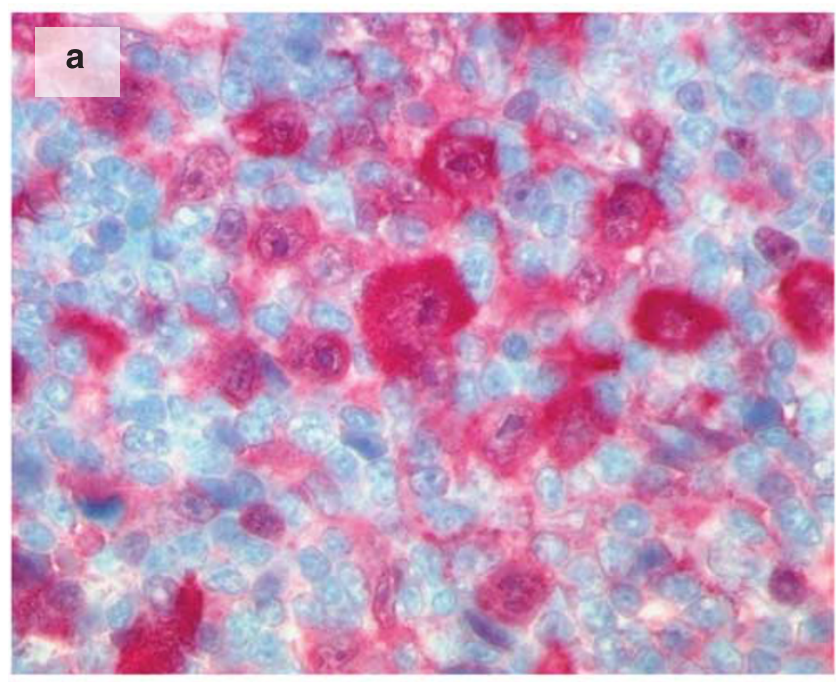

\section{Transient Transfection of Galectin-3 to HEK-293 Cells}

We transiently transfected myc-DDK-tagged galectin-3 cDNA plasmid (pCMV6 Entry vector, Origene, No. RC208785) to the cell line HEK-293. Transfection was performed by Turbofect (Fermentas, Life Sciences, St Leon-Rot, Germany) according to the manufacturer's instructions. Galectin-3 expression was validated by flow cytometry.

Native and mock-transfected HEK-293 cells did not show galectin-3 expression. Galectin-3 expression and morphology of transiently transfected HEK-293 cells were validated at 48 and $72 \mathrm{~h}$ of transfection by immunoblot and phase-contrast microscopy, respectively.

\section{Statistical Analysis}

Statistical analysis was performed with Student's $t$-test or $\chi^{2}$-test. $P$-values of $<0.05$ were considered to be statistically significant.
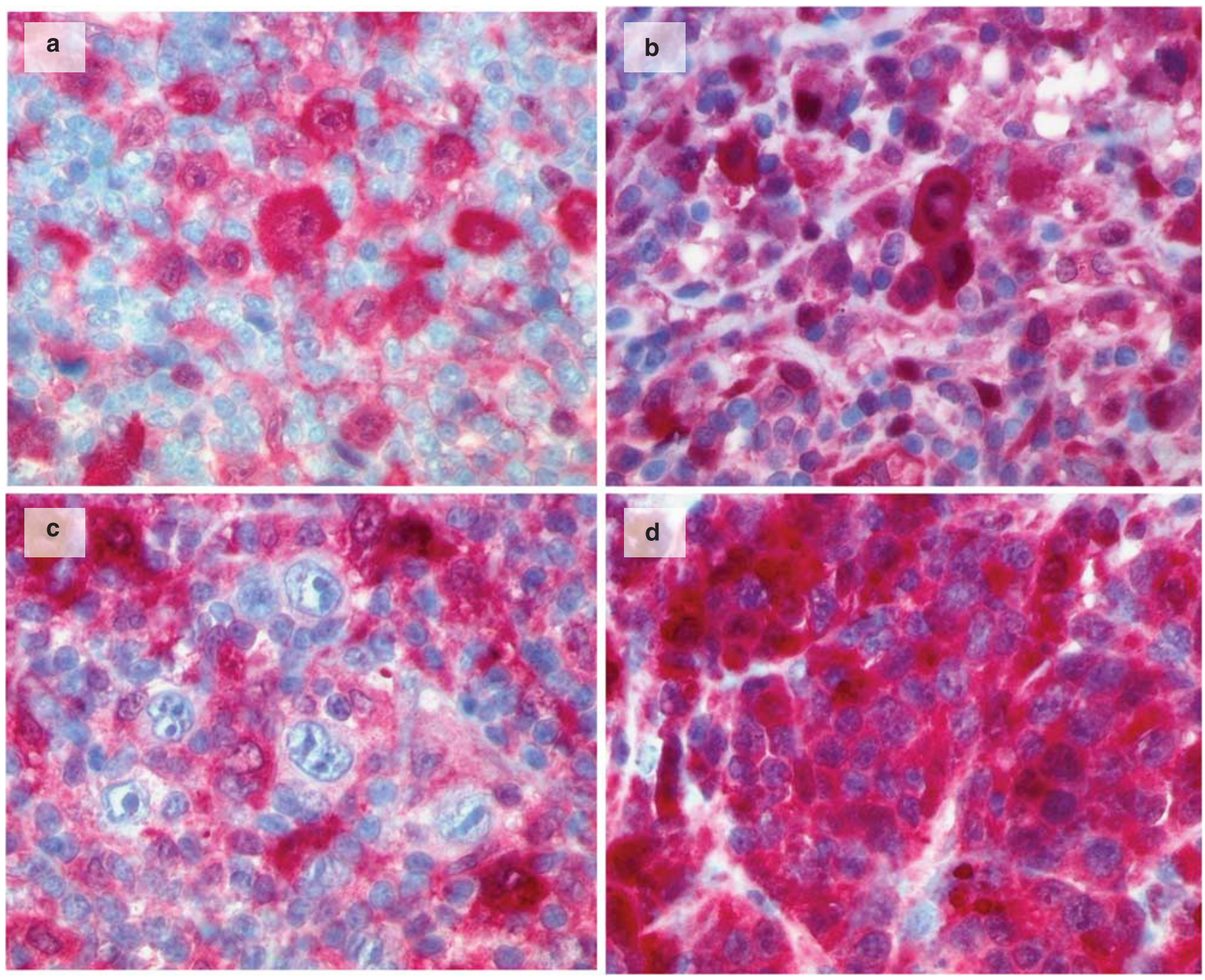

Figure 1 TMA analysis of HL and ALCL. (a) Galectin-1 is expressed in the cytoplasm of Reed-Sternberg (RS) cells (original magnification $\times 600$ ). (b) Galectin-1 is expressed in the cytoplasm of ALCL cells (original magnification $\times 600$ ). (c) Galectin-3 is not expressed in RS cells, but expressed in the surrounding macrophages (original magnification $\times 600$ ). (d) Galectin-3 is expressed in the cytoplasm of ALCL lymphoma cells (original magnification $\times 600$ ). 


\section{RESULTS}

Immunohistological Analysis of Galectin-1 and Galectin-3 of HL and ALCL Cases and Cell Lines by TMA-Staining

Galectin-1 is expressed in all cell lines of HL and ALCL. There is no significant difference of galectin-1 expression between ALK-positive and ALK-negative ALCL cell lines. The galectin-3 expression is heterogeneous between HL and ALCL cell lines. The analysis of 6 cases of cHL and 19 cases of ALCL revealed that galectin- 1 is expressed in all cases, except one cHL case. Galectin-1 is detected in the cytoplasm of cHL (HRS cells; Figure 1a) and ALCL tumor cells by immunohistology (Figure 1b). On the other hand, all cases of HL are galectin-3 negative (Figure 1c) and almost all ALCL cases were galectin-3 positive (Figure 1d). There is no difference in galectin-3 expression between ALK-positive and ALK-negative ALCL cases (Table 1). Galectin-3 expression is significantly different in CHL and ALCL $\left(\chi^{2}\right.$-test, ${ }^{\star} P=0.0000042$, Table 1$)$.

\section{Galectin-1-Induced Cell Death}

Galectin-1 treatment $(5 \mu \mathrm{M})$ for $24 \mathrm{~h}$ induces cell death in the HL cell lines KMH2 (47\%) as well as in the ALCL cell lines Karpas 299 (40\%) and SR786 (79\%) (Figure 2a), but in L428 only $3 \%$ of all cells suggesting that L428 is tolerant to galectin-1-induced cell death. There is heterogeneity of galectin-1-induced cell death among cell lines (Figure 2b). At $48 \mathrm{~h}$ of galectin-1 treatment, almost all cell lines underwent massive cell death (data not shown).

\section{Influence of CD30 Pre-Stimulation on Galectin-1-Induced Cell Death}

Galectin-1-induced cell death is enhanced by CD30 pre-stimulation of Karpas 299 (Figure 3a). Galectin-1-induced cell slightly increase by CD30 pre-stimulation of DEL, but it is not statistically significant (Figure 3b) and JB6 (Figure 3c). Galectin-1 could not induce cell death in combination with CD30 pre-stimulation of L428 (Figure 3d) and galectin-1 could not be influenced by CD30 pre-stimulation of L1236 cells (Figure 3e). CD30 pre-stimulation slightly decreases galectin-1-induced cell death in $\mathrm{KMH} 2$ (Figure 3f) and HDLM2 cells (Figure 3g), but there is no significant.

\section{Caspase Inhibition Completely Inhibited Galectin-1- Induced Cell Death Whereas a Significant Proportion of CD30/Galectin-1-Induced Cell Death Remained Unaffected}

Galectin-1-induced cell death was significantly inhibited by the pan-caspase inhibitor z-VAD-fmk in Karpas 299 cells. CD30-mediated enhancement of galectin-1-induced cell death was significantly inhibited by z-VAD-fmk, ie, cell death decreased from 78 to $20 \%$, where about $10-15 \%$ of cell death remained unaffected by caspase inhibition (Figure $3 \mathrm{~h}$ ).

TRAF1, TRAF2, and CIAP2 Expression of CD30Stimulated and Galectin-1-Treated Karpas 299 Cells

Consistent with published data, CD30 stimulation increased the expression of TRAF1 and cIAP2 but decreased the expression of TRAF2 at $24 \mathrm{~h}$. Galectin- 1 treatment for $6 \mathrm{~h}$ did

Table 1 Galectin-1 and galactin-3 expression in ALCL and HL

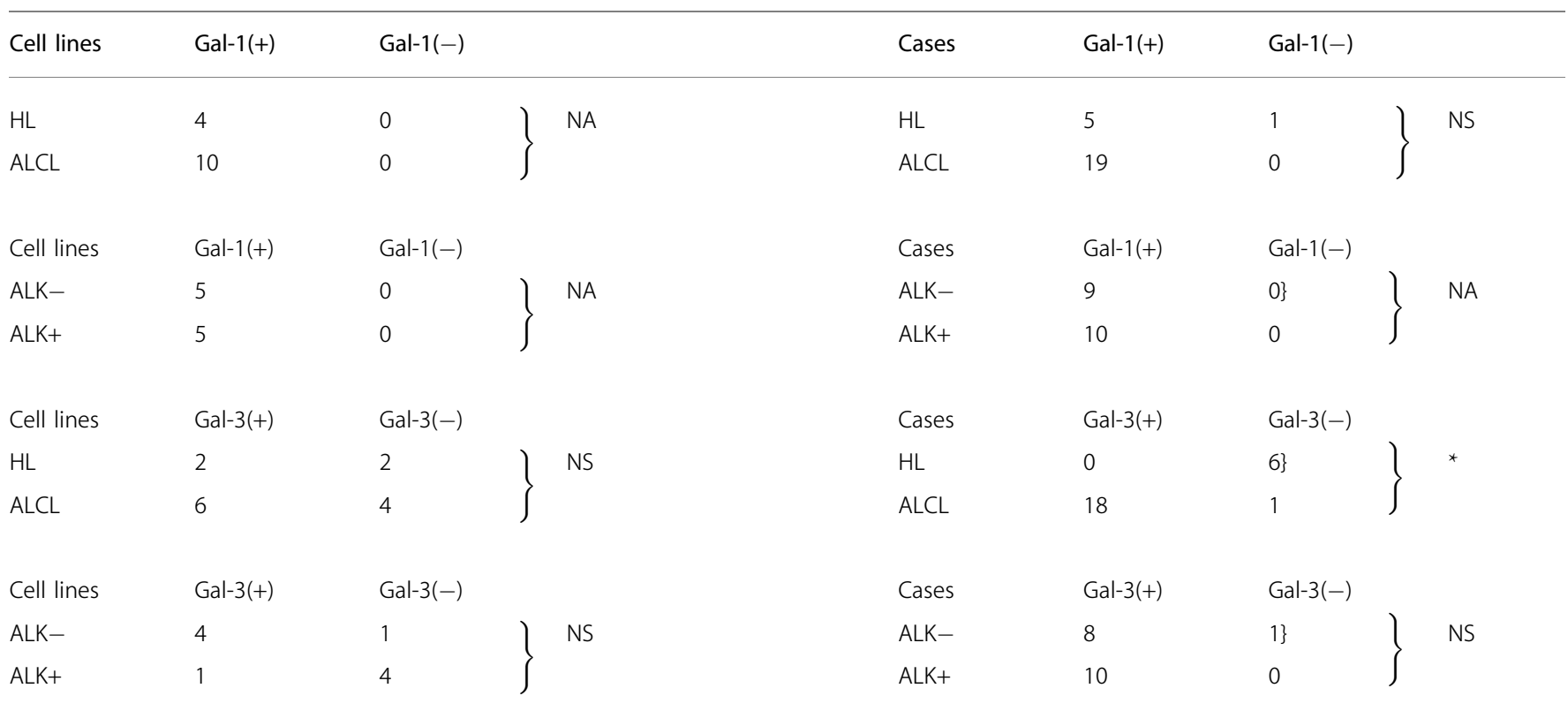

Expression of galectin- 1 and galectin-3 in $\mathrm{HL}$ and ALCL $\left({ }^{*} P=0.0000024, \mathrm{NS}\right.$ : not significant; NA: not applicable. The statistical significance is calculated by $\chi^{2}$ test. HL: Hodgkin lymphoma; ALCL: anaplastic large cell lymphoma; gal-1: galectin-1/galaptin; gal-3: galectin-3). 
a

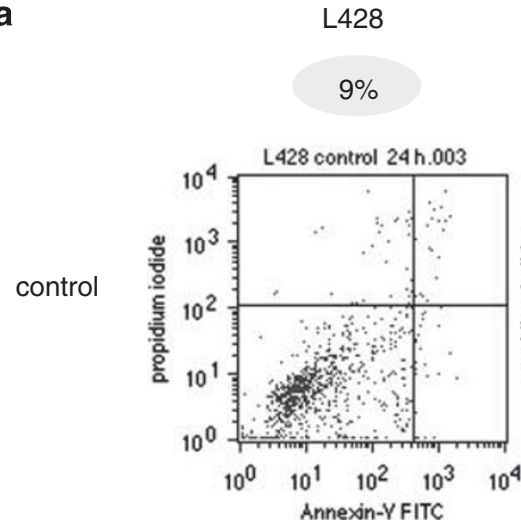

$12 \%$

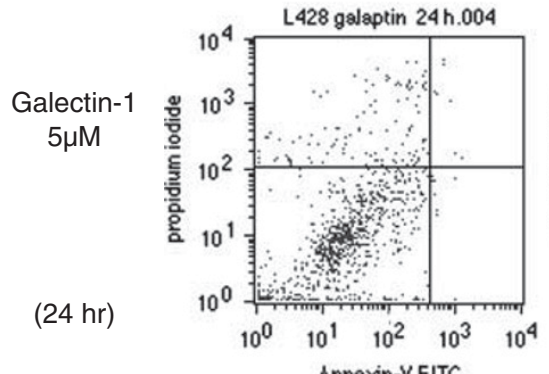

$\mathrm{KMH} 2$

$19 \%$

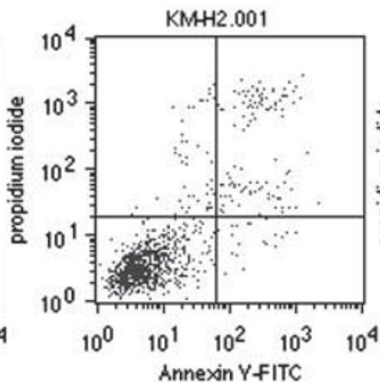

$66 \%$

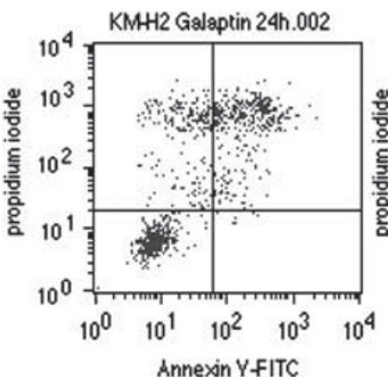

Karpas299

$9 \%$

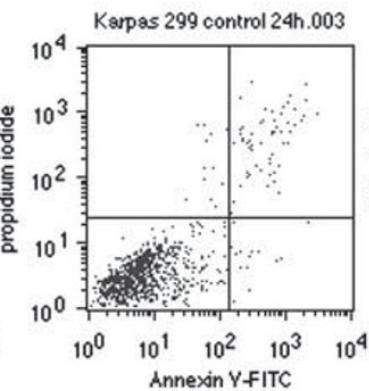

$49 \%$

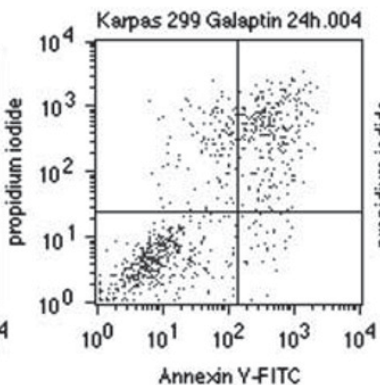

SR786

$19 \%$

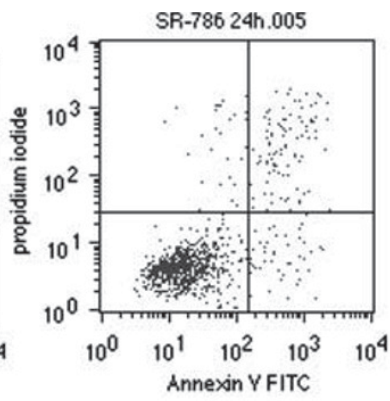

$98 \%$

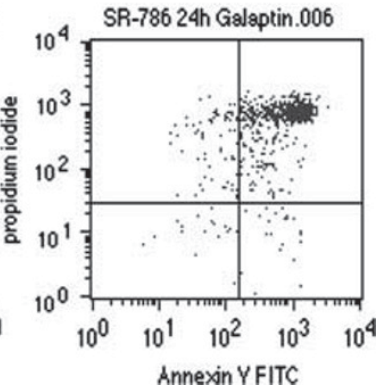

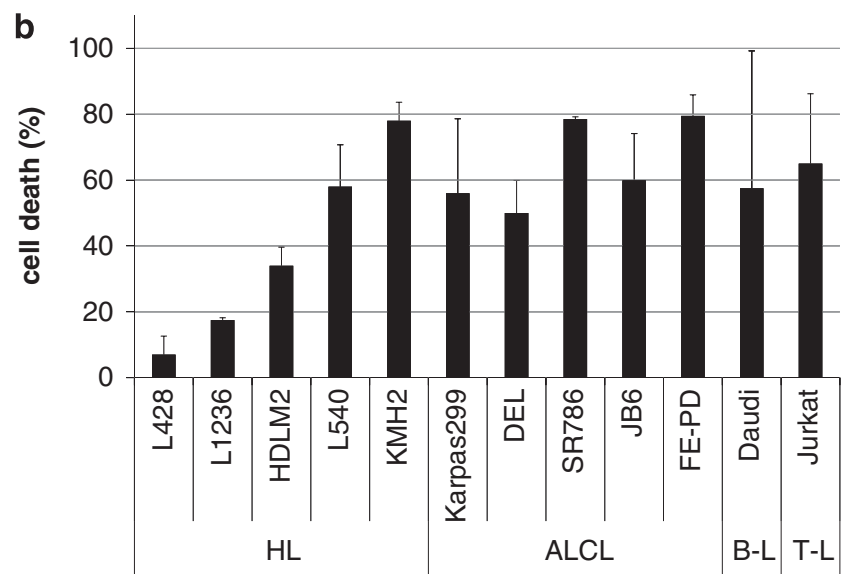

Figure 2 Galectin-1-induced cell death. (a) Flow-cytometric analysis of $5 \mu \mathrm{M}$ galectin-1-induced cell death at 24h in ALCL (Karpas 299 and SR786) and $\mathrm{HL}$ (L428 and KNH2) cell lines. The percentage of dead cells (Annexin V-FITC and PI positive) is indicated. (b) Flow-cytometric analysis of apoptosis mediated by $5 \mu \mathrm{M}$ galectin-1 treatment of ALCL cell line (Karpas 299, DEL, SR786, JB6, and FE-PD), HL cell line (L1236, L428, KMH2, HDLM2, and L540) and a T (Jurkat), and B-cell line (Daudi) for $24 \mathrm{~h}$. Constitutive cell death revealed by Tris buffer treatment is subtracted. Data represent an average \pm s.d. from two independent experiments.

not influence CD30-induced TRAF1/cIAP2 expression or CD30-mediated TRAF2 degradation (Figure 4).

\section{The Effect of Galectin-3 Knockdown on Galectin-1-Induced Cell Death}

Galectin-3 siRNA transfection of Karpas 299 cells significantly reduced mRNA expression, as analyzed by RT-RQPCR at $24 \mathrm{~h}$ after transfection (Figure $5 \mathrm{a}$ ). Galectin-3 protein expression was significantly inhibited by galectin- 3 siRNA, as revealed by western blot analysis. Knockdown of galectin-3 by siRNA inhibits the expression of galectin-3 as compared with control siRNA-treated cells at $48 \mathrm{~h}$ of transfection (Figure 5b). Galectin-3 siRNA-mediated knockdown did not alter galectin-1- or CD30/galectin-1-induced cell death as compared with control siRNA-transfected cells (Figure 5c).

\section{Galectin-3 Expression by Transient Transfected HEK-293 Cells}

Based on western blot analysis, HEK-293 cells are galectin-3 negative. We transiently transfected HEK-293 cells with a 
a

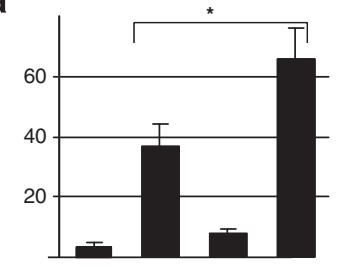

C

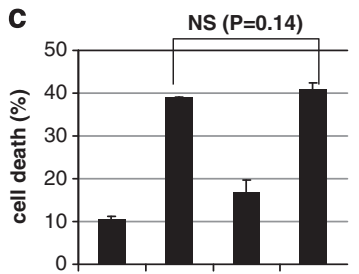

e

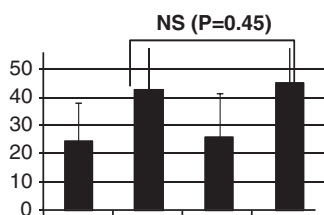

9

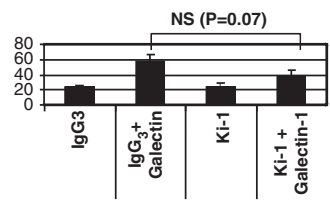

b

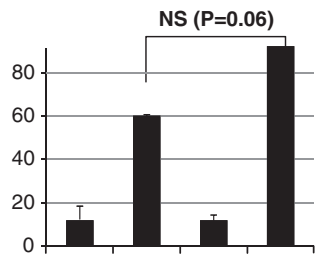

d

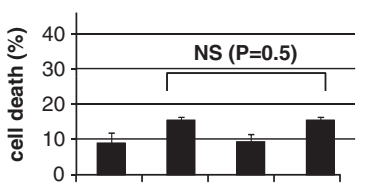

$\mathbf{f}$

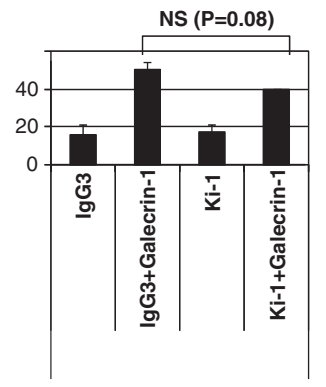

h

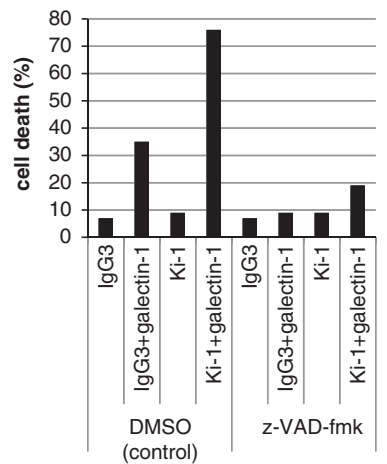

Figure 3 CD30-mediated influence of galectin-1-mediated cell death and caspase inhibition. The vertical line represents the percentage of cell death. $P$-values were calculated by Student's $t$-test. Data represent an average \pm s.d. from two independent experiments. (a) Galectin-1-induced cell death was enhanced by CD30 pre-stimulation (Ki-1) of Karpas 299 cells ( $\left.{ }^{*} P=0.044604\right)$. (b) Galectin-1-induced cell death tended to be enhanced by CD30 prestimulation of DEL cells $(P=0.06290)$. (c) CD30 pre-stimulation slightly induced cell death in JB6, galectin-1-induced cell death was not changed by CD30 stimulation $(P=0.14758)$. (d-g) The galectin-1-induced cell death was not changed by CD30 pre-stimulation of L428 and L1236 cells ( $(\mathbf{d})$ L428, $P=0.5$, (e) L1236, $P=0.452588)$ and tended to decrease in KMH2 and HDLM2 in combination with CD30 pre-stimulation $((\mathbf{f}) \mathrm{KMH} 2, P=0.084490$, (g) HDLM2, $P=0.07125$ ). But there is no statistically significant. NS: not significant. (h) Galectin-1-induced cell death was completely inhibited by the pan-caspase inhibitor Z-VAD-fmk in Karpas 299 cells. Noteworthy, apoptosis of CD30/galectin-1-treated Karpas 299 cells was only partially inhibited by z-VAD-fmk. In all, 10-15\% of cell death remained unaffected by caspase inhibition. One out of two experiments is shown.

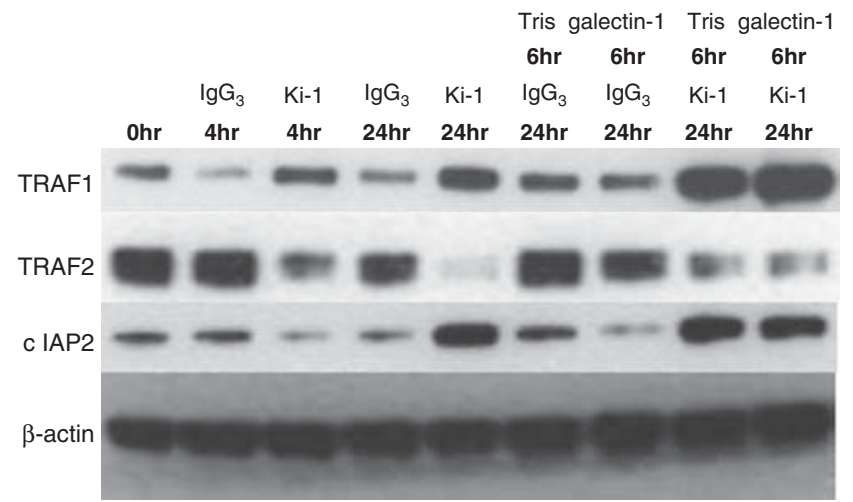

Figure 4 TRAF1, TRAF2, and CIAP2 expression of galectin-1 and CD30stimulated Karpas 299 cells. TRAF1 and cIAP2 expression was increased and TRAF2 expression was decreased by CD30 stimulation at $24 \mathrm{~h}$ as compared with $\mathrm{IgG}_{3}$ treatment. Galectin-1/galaptin treatment did not influence CD30mediated changes of TRAF1, TRAF2, or CIAP2 expression. A representative western blot is shown (one out of three). galectin-3 expression plasmid. Forty-eight hours after transfection, cells expressed galectin-3 on a high level, as revealed by flow-cytometric (Figure 6a) and western blot analysis (Figure 6b). Mock-transfected cells remained galectin-3 negative. Galectin-3 expression remained on a high level even at $72 \mathrm{~h}$ of transfection (data not shown).

\section{Morphology of Galectin-3 Transfected HEK-293 Cells}

Forty-eight hours after galectin-3 transfection, morphology of HEK-293 cells was evaluated by phase-contrast microscopy. Native or mock-transfected HEK-293 cells adhered to the culture plate and showed regular spreading. There was no sign of cell aggregation in native and mocktransfected cells (Figure 6cI and cII) while galectin-3-transfected HEK-293 cells showed massive cell aggregation with almost complete loss of adherence and spreading (Figure 6cIII). 
a

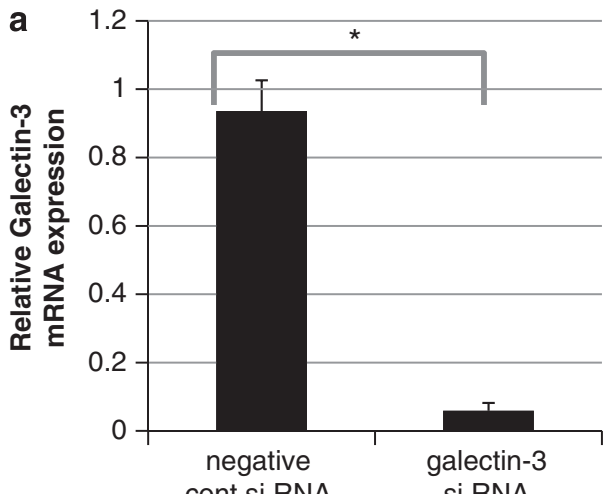

b

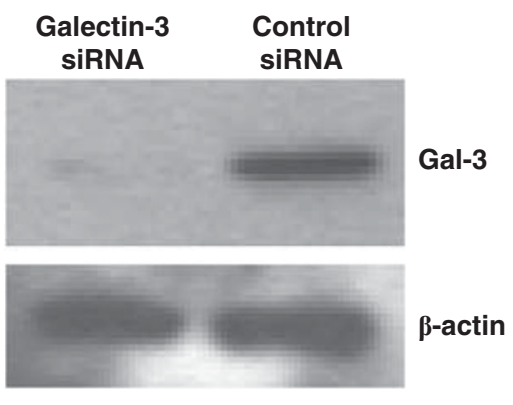

C NS

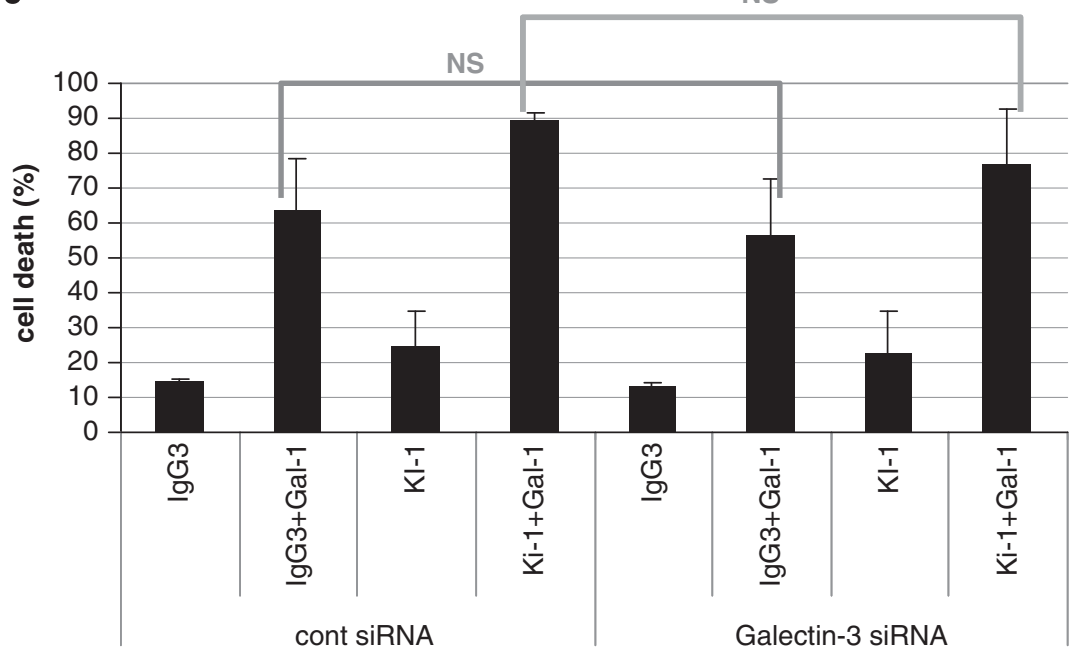

Figure 5 Knockdown of galectin-3 by siRNA transfection and galectin-1-induced cell death. (a) Galectin-3 siRNA treatment for $24 \mathrm{~h}$ significantly inhibited galectin-3 mRNA expression, as revealed by real-time RT-PCR analysis. HPRT-normalized relative expression of galectin-3 mRNA is indicated. ${ }^{\star} P=0.00108$, the $P$-value was calculated by Student's $t$-test. Data represent an average \pm s.d. from three independent experiments. (b) Western blot analysis of galectin-3 siRNA-mediated knockdown of galectin-3 expression at $48 \mathrm{~h}$ of transfection (Galectin-3 runs at about $30 \mathrm{kDa}$ ). $\beta$-Actin was used as endogenous control ( $\beta$-actin runs at about $40 \mathrm{kDa}$ ). Depicted data are representative from two independent experiments. (c) Based on flow-cytometric analysis, CD30-mediated enhancement of galectin-1-induced cell death was not altered by galectin-3 expression. Knockdown of galectin-3 by siRNA could not alter the amount of galectin-1-induced cell death with or without CD30 stimulation of Karpas 299 cells siRNA (NS: not significant). The statistical significant was determined by Student's $t$-test. Data represent an average of two independent experiments and bars represent standard deviation.

\section{DISCUSSION}

We previously reported that galectin-1 can induce cell death in DLBCL, ${ }^{7}$ suggesting that endogenous lectin might be a regulator of cell death in this lymphoma entity. Here, we demonstrate that galectin-1 can induce cell death in HL and ALCL cell lines as well. Therefore, galectin-1 might exhibit a universal role in the induction of cell death, regardless of histological subtypes of human malignant lymphoma.

Stein et al. ${ }^{16}$ described the CD30 antigen to be expressed in reactive and neoplastic lymphoid tissues. Dürkop et al. ${ }^{17}$ revealed that $\mathrm{CD} 30$ belongs to the TNFR superfamily. Hübinger et al. ${ }^{18}$ reported that the cellular inhibitors of apoptosis proteins, eg, cIAP1 and cIAP2 are induced by CD30 stimulation in ALCL cells. Hirsch et al. ${ }^{8}$ reported that CD30-induced signaling is absent in HL cells but present in ALCL cells. Hübinger et al. ${ }^{19}$ and Hirsch et al. ${ }^{8}$ revealed that CD30 stimulation did not induce apoptosis in ALCL cell lines. In the present study, we demonstrated that galectin-1induced cell death is significantly increased by CD30 prestimulation of ALCL cells. These data suggest an interference of galectin-1 and CD30 signaling, where a caspase-dependent (galectin-1 mediated) and to some extent a caspase-independent pathway (CD30 signaling, Figure $3 \mathrm{~h}$ ) is activated to induce cell death. The $\mathrm{CD} 30$ pre-stimulation is not effective in all HL and some ALCL cell lines. In L428, L1236, KMH2, HDLM2, DEL, and JB6 cells, galectin-1-induced cell death was not significantly altered by CD30 pre-stimulation. Published data demonstrate heterogeneous effects of CD30 stimulation in different lymphoma cell lines. ${ }^{20}$ Hirsch et al. ${ }^{8}$ reported that CD30 is effective in ALCL, but not in HL. Therefore, different effects of galectin-1-induced cell death and the influence of CD30 signaling in different lymphoma cell lines are not surprising. CD30 signaling positively influences galectin-1-induced cell death in ALCL cell line, 


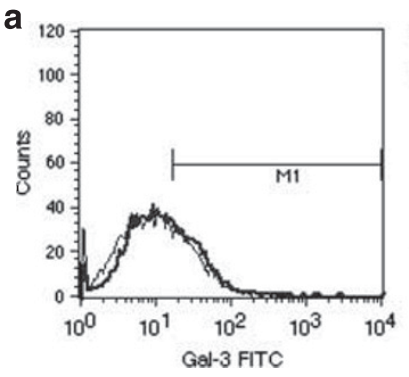

$5.5 \%$

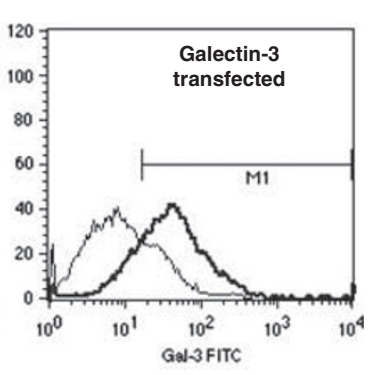

$49.5 \%$

b

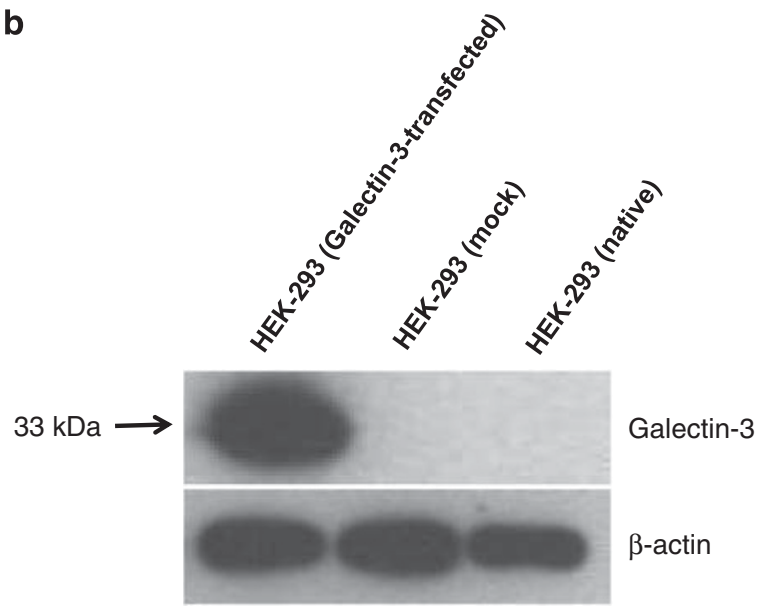

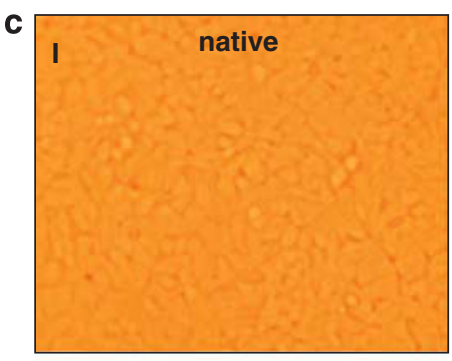

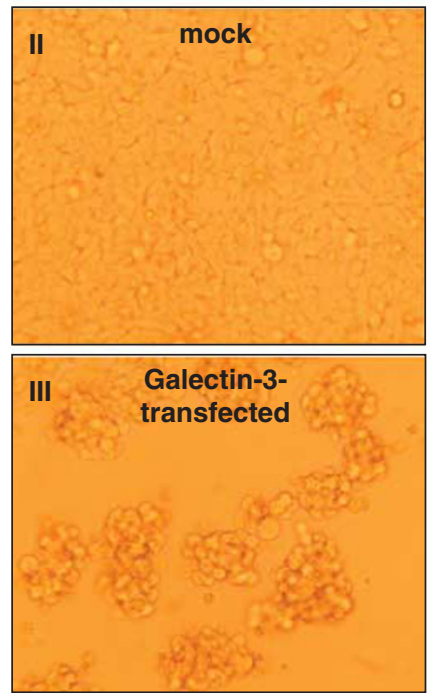

Figure 6 Galectin-3 expression and function. (a) Galectin-3 transfection of HEK-293 cells leads to galectin-3 cell surface expression ( 49.5\%). Native or mock-transfected HEK-293 cells were galectin-3 negative. The thin line represents the isotype control Ab and the thick line represents galectin-3 Ab. The percentage of galectin-3 expression is indicated. Representative data out of two independent experiments are shown. (b) Western blot analysis of galectin-3 expression. Native or mock-transfected HEK-293 cells do not express galectin-3. Galectin-3 transfection of HEK-293 cells leads to massive galectin-3 expression. $\beta$-Actin served as loading control $(40 \mathrm{kDa})$. A representative western blot is shown out of two independent experiments. (c) Galectin-3-mediated changes of cell morphology. At $48 \mathrm{~h}$ of galectin-3 transfection to HEK-293 cells, the cell morphology was evaluated by phase-contrast microscopy. (I) Native HEK-293 cells showed spreading and adhered to the surface of the culture plate. No cell aggregation occurred. (II) Mock-transfected HEK-293 cells appeared like native HEK-293 cells. (III) Galectin-3 transfected HEK-293 cells showed massive cell aggregation. Original magnification is $\times 200$. Representative data form one out of five independent experiments are shown.

Karpas 299. This CD30 effect is not present in HL cell lines, which is concordant with published data, where Hodgkin cell lines are shown to be explained by the fact that there is no CD30 signaling in Hodgkin cell lines. ${ }^{8}$ In ALCL cell lines, DEL and JB6, CD30 stimulation could not significantly enhance galectin-1-induced cell death. While CD30 can enhance galectin-1-induced cell death in Karpas 299, the reason why CD30 could not enhance galectin-1-induced cell death in DEL and JB6 is under future investigation.

In order to clarify the interaction between galectin-1-induced cell death and CD30 signaling, we focused on the cell line Karpas 299. CD30 belongs to the subclass of the TNFR family without a death domain. Instead, adaptor molecules like TRAF1, TRAF2, TRAF3, and TRAF5 interact with the cytoplasmic domain of CD30 to transmit the signal. ${ }^{21-23}$ TRAF1 is an anti-apoptotic molecule of the intracellular signaling pathway of TNFR family, the expression of which is induced by $\mathrm{CD} 30$ stimulation. ${ }^{24}$ We confirmed that CD30 stimulation induced TRAF1 expression in Karpas 299, and in the present study we revealed that galectin-1 treatment did not influence this regulation (Figure 4). The CD30mediated downregulation of TRAF2 is known to be a mechanism which negatively regulates CD30-induced NF- $\kappa \mathrm{B}$ activation. ${ }^{25}$ Our experiments revealed that CD30-mediated TRAF2 downregulation is not affected by galectin-1 treatment in Karpas 299 cells. cIAP2 is highly expressed in HRS cells and inhibits apoptosis by interfering with constitutively active caspase-3. CD30 stimulation induces cIAP2 expression through NF- $\kappa \mathrm{B}$ activation in ALCL cells. ${ }^{26}$ Our study demonstrates that galectin-1 treatment did not influence CD30-mediated cIAP2 induction. Therefore, a TRAF1, TRAF2, and cIAP2-independent pathway is activated by CD30 stimulation triggering the enhancement of galectin-1induced cell death, which is to some extent caspase independent. CD45RO is known to be a receptor for galectin- $1 .{ }^{27}$ According to our preliminary analysis, CD45RO expression of HL and ALCL cell lines is closely correlated with the sensitivity to galectin-1 (data not shown). Therefore, we assume that CD45RO is a receptor for galectin-1 in HL and ALCL cell lines. 
It is reported that galectin-3 represents a useful marker for differentiation between HL and ALCL. ${ }^{9}$ This could be confirmed by our study. Galectin-3 is known to have anti-apoptotic functions in various tumors. ${ }^{11}$ The expression of galectin-3 might be crucial for the biological differences of ALCL and HL. Galectin-3 is known to influence galectin-1 function, ie, can inhibit galectin-1-induced apoptosis. ${ }^{14}$ However, in Karpas 299 cells, galectin-3 knockdown neither changed galectin-1-induced cell death per se nor CD30/galectin-1-induced cell death significantly (Figure 5c). Therefore, at least in Karpas 299 cells, galectin-3 does not influence galectin-1 effects.

Cell surface galectin-3 mediates homotypic cell adhesion and aggregation of tumor cells. ${ }^{28}$ In order to clarify the biological function of galectin-3, we transfected galectin-3 cDNA to HEK-293 cells. Native and mock-transfected HEK-293 cells did not show any cell aggregation or adherence to culture plates. Of note, galectin-3 transfection induced complete cell aggregation of HEK-293 cells (Figure 6c). These findings demonstrate that galectin-3 is involved in cell-to-cell interactions and acts as a cell adhesion molecule.

In summary, this is the first report investigating the influencing effect of CD30 signaling on galectin-1-induced cell death. This effect is heterogenous between HL and ALCL cell lines, where galectin-1 consistently induced cell death. Our data suggest that CD30-targeted therapy in combination with galectin-1 treatment might induce effective killing of ALCL cells, whereas HL cells are not susceptible to this combined treatment. Finally, the function of galectin-3 as an adhesion molecule, associated with cell-to-cell interactions, is substantiated by this work.

\section{ACKNOWLEDGEMENTS}

We are grateful to E von der Wall, I Puschendorf, and C Cieluch for excellent technical assistance and $\operatorname{Dr} L$ Dimitrova for advice.

\section{DISCLOSURE/CONFLICT OF INTEREST}

The authors declare no conflict of interest.

1. Rabinovich GA, Rubinstein N, Toscano MA. Role of galectins in inflammatory and immunomodulatory processes. Biochim Biophys Acta 2002;1572:274-284.

2. Barondes SH. Soluble lectins: a new class of extracellular proteins. Science 1984;223:1259-1264.

3. Adams L, Scott GK, Weinberg CS. Biphasic modulation of cell growth by recombinant human galectin-1. Biochim Biophys Acta 1996;1312:137-144.

4. Perillo NL, Pace KE, Seilhamer JJ, et al. Apoptosis of T cells mediated by galectin-1. Nature 1995;378:736-739.

5. Rodig SJ, Ouyang J, Juszczynski P, et al. AP1-dependent galectin-1 expression delineates classical Hodgkin and anaplastic large cell lymphomas from other lymphoid malignancies with shared molecular features. Clin Cancer Res 2008;14:3338-3344.

6. Juszczynski P, Ouyang J, Monti S, et al. The AP1-dependent secretion of galectin-1 by Reed Sternberg cells fosters immune privilege in classical Hodgkin lymphoma. Proc Natl Acad Sci USA 2007;104: 13134-13139.

7. Suzuki O, Nozawa Y, Abe M. Regulatory roles of altered $\mathrm{N}$ - and O-glycosylation of CD45 in galectin-1-induced cell death in human diffuse large B cell lymphoma. Int J Oncol 2005;26:1063-1068.
8. Hirsch B, Hummel M, Bentink S, et al. CD30-induced signaling is absent in Hodgkin's cells but present in anaplastic large cell lymphoma cells. Am J Pathol 2008;172:510-520.

9. Konstantinov KN, Robbins BA, Liu FT. Galectin-3, a beta-galactosidebinding animal lectin, is a marker of anaplastic large-cell lymphoma. Am J Pathol 1996;148:25-30.

10. Hoyer KK, Pang M, Gui D, et al. An anti-apoptotic role for galectin-3 in diffuse large B-cell lymphomas. Am J Pathol 2004;164: 893-902.

11. Akahani S, Nangia-Makker $\mathrm{P}$, Inohara $\mathrm{H}$, et al. Galectin-3: a novel antiapoptotic molecule with a functional $\mathrm{BH} 1$ (NWGR) domain of $\mathrm{Bcl}-2$ family. Cancer Res 1997;57:5272-5276.

12. Fukumori T, Takenaka Y, Yoshii T, et al. CD29 and CD7 mediate galectin-3-induced type II T-cell apoptosis. Cancer Res 2003;63: 8302-8311.

13. Suzuki O, Abe M. Cell surface N-glycosylation and sialylation regulates galectin-3-induced apoptosis in human diffuse large B cell lymphoma. Oncol Rep 2008;19:743-748.

14. Hahn HP, Pang M, He J, et al. Galectin-1 induces nuclear translocation of endonuclease $\mathrm{G}$ in caspase- and cytochrome c-independent $\mathrm{T}$ cell death. Cell Death Diff 2004;11:1277-1286.

15. Livak KJ, Schmittgen TD. Analysis of relative gene expression data using real-time quantitative PCR and the 2(-Delta Delta C(T)) method. Methods 2001;25:402-408.

16. Stein H, Mason DY, Gerdes J, et al. The expression of the Hodgkin's disease associated antigen $\mathrm{Ki}-1$ in reactive and neoplastic lymphoid tissue: evidence that Reed-Sternberg cells and histiocytic malignancies are derived from activated lymphoid cells. Blood 1985;66:848-858.

17. Dürkop $H$, Latza $U$, Hummel $M$, et al. Molecular cloning and expression of a new member of the nerve growth factor receptor family that is characteristic for Hodgkin's disease. Cell 1992;68: 421-427.

18. Hübinger G, Schneider C, Stöhr D, et al. CD30-induced up-regulation of the inhibitor of apoptosis genes CIAP1 and CIAP2 in anaplastic large cell lymphoma cells. Exp Hematol 2004;32:382-389.

19. Hubinger G, Muller E, Scheffrahn I, et al. CD30-mediated cell cycle arrest associated with induced expression of p21(CIP1/WAF1) in the anaplastic large cell lymphoma cell line Karpas 299. Oncogene 2001;20:590-598.

20. Jung W, Krueger S, Renner C, et al. Opposite effects of the CD30 ligand are not due to CD30 mutations: results from cDNA cloning and sequence comparison of the CD30 antigen from different sources. Mol Immunol 1994;31:1329-1334.

21. Gedrich RW, Gilfillan MC, Duckett CS, et al. CD30 contains two binding sites with different specificities for members of the tumor necrosis factor receptor-associated factor family of signal transducing proteins. J Biol Chem 1996;271:12852-12858.

22. Lee SY, Park CG, Choi Y. T cell receptor-dependent cell death of T cell hybridomas mediated by the CD30 cytoplasmic domain in association with tumor necrosis factor receptor-associated factors. J Exp Med 1996;183:669-674.

23. Aizawa $\mathrm{S}$, Nakano $\mathrm{H}$, Ishida $\mathrm{T}$, et al. Tumor necrosis factor receptorassociated factor (TRAF) 5 and TRAF2 are involved in CD30-mediated NFkappaB activation. J Biol Chem 1997;272:2042-2045.

24. Dürkop $\mathrm{H}$, Hirsch $\mathrm{B}$, Hahn $\mathrm{C}$, et al. Differential expression and function of A20 and TRAF1 in Hodgkin lymphoma and anaplastic large cell lymphoma and their induction by CD30 stimulation. J Pathol 2003;200:229-239.

25. Duckett CS, Thompson CB. CD30-dependent degradation of TRAF2: implications for negative regulation of TRAF2 signaling and the control of cell survival. Genes Dev 1997;11:2810-2821.

26. Dürkop $\mathrm{H}$, Hirsch $\mathrm{B}$, Hahn $\mathrm{C}$, et al. clAP2 is highly expressed in Hodgkin-Reed-Sternberg cells and inhibits apoptosis by interfering with constitutively active caspase-3. J Mol Med 2006;84: 132-141.

27. Pace $\mathrm{KE}$, Lee $\mathrm{C}$, Stewart $\mathrm{PL}$, et al. Restricted receptor segregation into membrane microdomains occurs on human $T$ cells during apoptosis induced by galectin-1. J Immunol 1999;163:3801-3811.

28. Dumic J, Dabelic S, Flogel M. Galectin-3: an open-ended story. Biochim Biophys Acta 2006;1760:616-635. 